\title{
Unilateral triple mandibular canal with double mandibular foramen: cone-beam computed tomography findings of an unexpected anatomical variant
}

\author{
A. Borghesi(D, M.P. Bondioni \\ Department of Medical and Surgical Specialties, Radiological Sciences and Public Health, University of Brescia, \\ ASST Spedali Civili of Brescia, Italy
}

[Received: 7 May 2020; Accepted: 17 May 2020]

The mandibular canal is a bony channel located within the spongiosa of the mandible. The main structure contained in the mandibular canal is the inferior alveolar nerve. The inferior alveolar nerve is a very important structure that requires due consideration during dental or surgical procedures involving the mandible. Therefore, a detailed morphological analysis of the mandibular canal should be carried out before any surgical procedure in the mandibular region in order to avoid complications and to reduce the risk of inadequate local nerve blocking. The human mandible typically has a single mandibular canal on each side; however, accessory mandibular canals have been described previously in the literature. The most common variant of the mandibular canal is the bifid mandibular canal, which has a prevalence ranging from $10 \%$ to $66 \%$ on cone-beam computed tomography (CBCT) examinations. A rare variant of bifid mandibular canal is the trifid canal, accounting for less than $6 \%$ of all bifid canals. In some cases, the bifid and trifid mandibular canals are associated with a double mandibular foramen, which is a rare anatomical variant with a reported incidence of $1.35 \%$ on CBCT images. Herein, we present the interesting CBCT images of an unexpected anatomical variant characterised by unilateral triple mandibular canal with double mandibular foramen in a young Caucasian woman. (Folia Morphol 2021; 80, 2: 471-475)

Key words: mandibular canal, anatomic variation, accessory mandibular canal, accessory mandibular foramen, mandible, cone-beam computed tomography

\section{INTRODUCTION}

The mandibular canal is a bony channel located within the spongiosa of the mandible, typically close to the apices of the roots of the molars. The mandibular canal starts at the mandibular foramen (located on the lingual aspect of the mandibular ramus) and ends at the mental foramen (located on the buccal aspect of the mandibular body) [9]. It runs from the top to the bottom of the mandible, and forms an upward-facing curve.

The main structure contained in the mandibular canal is the inferior alveolar nerve (IAN). The IAN is

Address for correspondence: A. Borghesi, MD, Department of Medical and Surgical Specialties, Radiological Sciences and Public Health, University of Brescia, ASST Spedali Civili of Brescia, Piazzale Spedali Civili, 1, I-25123 Brescia, Italy, tel: +39 030395900, fax: +39 0303399897, e-mail: andrea.borghesi@unibs.it

This article is available in open access under Creative Common Attribution-Non-Commercial-No Derivatives 4.0 International (CC BY-NC-ND 4.0) license, allowing to download articles and share them with others as long as they credit the authors and the publisher, but without permission to change them in any way or use them commercially. 
the largest branch of the mandibular nerve, which is the third division of the fifth cranial nerve (the trigeminal nerve) [13]. Inside the mandibular canal, the IAN appears as a single large nerve bundle; however, it has been previously demonstrated that the IAN includes two distinct branches spirally twisted around each other [8]. Both nerves are wrapped in a layer of connective tissue (the perineurium). The larger branch corresponds to the mental nerve, whereas the smaller branch corresponds to the dental nerve $[8,13]$. The mental nerve comes out at the level of the mental foramen, while the dental nerve continues its intra-mandibular course beyond the mental foramen within the incisive mandibular canal [13].

The IAN is a very important structure that requires due consideration during dental or surgical procedures involving the mandible, such as dental implant placement, endodontic treatment, mandibular molar extractions (especially of the third molars), and periapical and orthognathic surgeries [8]. Therefore, a detailed morphological analysis of the mandibular canal should be carried out before any surgical procedure in the mandibular region in order to avoid complications, such as neurosensory impairment, traumatic neuroma, or bleeding, and to reduce the risk of inadequate local nerve blocking.

In the interest of contributing a novel finding to the literature, this report presents interesting cone-beam computed tomography (CBCT) images of an unexpected anatomical variant characterised by a triple mandibular canal with double mandibular foramen in the same hemimandible in a young Caucasian woman.

\section{CASE REPORT}

During a retrospective search on the department radiology information system/picture archiving and communication system between January 2012 and December 2019, we came across interesting CBCT images of a young Caucasian woman who underwent a CBCT scan of the mandible as part of her routine dental examination. The patient was in good health, and no systemic disease or hereditary syndromes were reported. The examination was carried out with high-quality CBCT scanner (NewTom Cone Beam 3D Imaging, Verona, Italy) using the following parameters: tube voltage $90 \mathrm{kVp}$, tube current $4 \mathrm{~mA}$, and field of view $10 \times 8 \mathrm{~cm}$. The CBCT acquisition data were reconstructed in the axial plane as thin-section images with a voxel size of $150 \mu \mathrm{m}$.
Unexpectedly, the CBCT images revealed an extremely rare anatomical variant characterised by a triple mandibular canal on the left side of the mandible (Figs. 1, 2). On the same hemimandible, a double mandibular foramen $\left(\mathrm{MF}_{1}\right.$ and $\left.\mathrm{MF}_{2}\right)$ was also observed (Figs. 1, 2). The maximum diameter of the left main mandibular canal $\left(\mathrm{MC}_{\text {main }}\right)$ was $2.6 \mathrm{~mm}$, and the maximum diameters of the two accessory mandibular canals $\left(\mathrm{AMC}_{1}\right.$ and $\mathrm{AMC}_{2}$ ) were $2 \mathrm{~mm}$ and $1.4 \mathrm{~mm}$, respectively.

The intra-mandibular position of $A M C_{1}$ and $A M C_{2}$ was in close proximity to $\mathrm{MC}_{\text {main }}$ (Fig. 1). $\mathrm{AMC}_{1}$ originated from $M C_{\text {main }}$ at the level of the second molar (Fig. 1). From its origin, $A M C_{1}$ ran forward in contact with the lingual cortex of the mandibular body, lingual to $M C_{\text {main }}$ (Fig. 1). $A M C_{1}$ joined $M C_{\text {main }}$ at the level of the mental foramen, continuing mesially into the incisive mandibular canal (Fig. 1). $\mathrm{AMC}_{2}$ originated from a small accessory mandibular foramen $\left(\mathrm{MF}_{2}\right)$ located inferior to the main mandibular foramen $\left(M_{1}\right)$ (Fig. 2). From its origin, $A M C_{2}$ ran forward and inferior to $M C_{\text {main }}$ (Figs. 1, 2). $A M C_{2}$ joined $M C_{\text {main }}$ at the level of the second premolar (Fig. 1).

With respect to the distomesial course of the triple mandibular canal, while the intra-mandibular course of $\mathrm{MC}_{\text {main }}$ and $\mathrm{AMC}_{2}$ formed an upward-facing curve (Fig. 2), the intra-mandibular course of $A M C_{1}$ showed a serpiginous shape in the anterior portion (Fig. 2).

\section{DISCUSSION}

The human mandible typically has a single mandibular canal on each side; however, accessory mandibular canals have been described previously in the literature $[6,10,12,14,15]$. The presence of accessory mandibular canals is likely due to the incomplete fusion of three distinct IANs that merge to form the IAN during the prenatal period [3].

The most common variant of the mandibular canal is the bifid mandibular canal, which has a prevalence ranging from $10 \%$ to $66 \%$ on CBCT examinations $[6,10,12,14,15]$. It is detected in the posterior mandible or mandibular ramus $[6,10,12,14,15]$. The most common and clinical important type of bifid mandibular canal is the retromolar canal $[6,10,12,14,15]$. A rare variant of bifid mandibular canal is the trifid canal, accounting for less than $6 \%$ of all bifid canals $[12,14]$.

In some cases, the bifid and trifid mandibular canals are associated with a double mandibular foramen [12], which is a rare anatomical variant with a reported incidence of $1.35 \%$ on CBCT images [4]. 


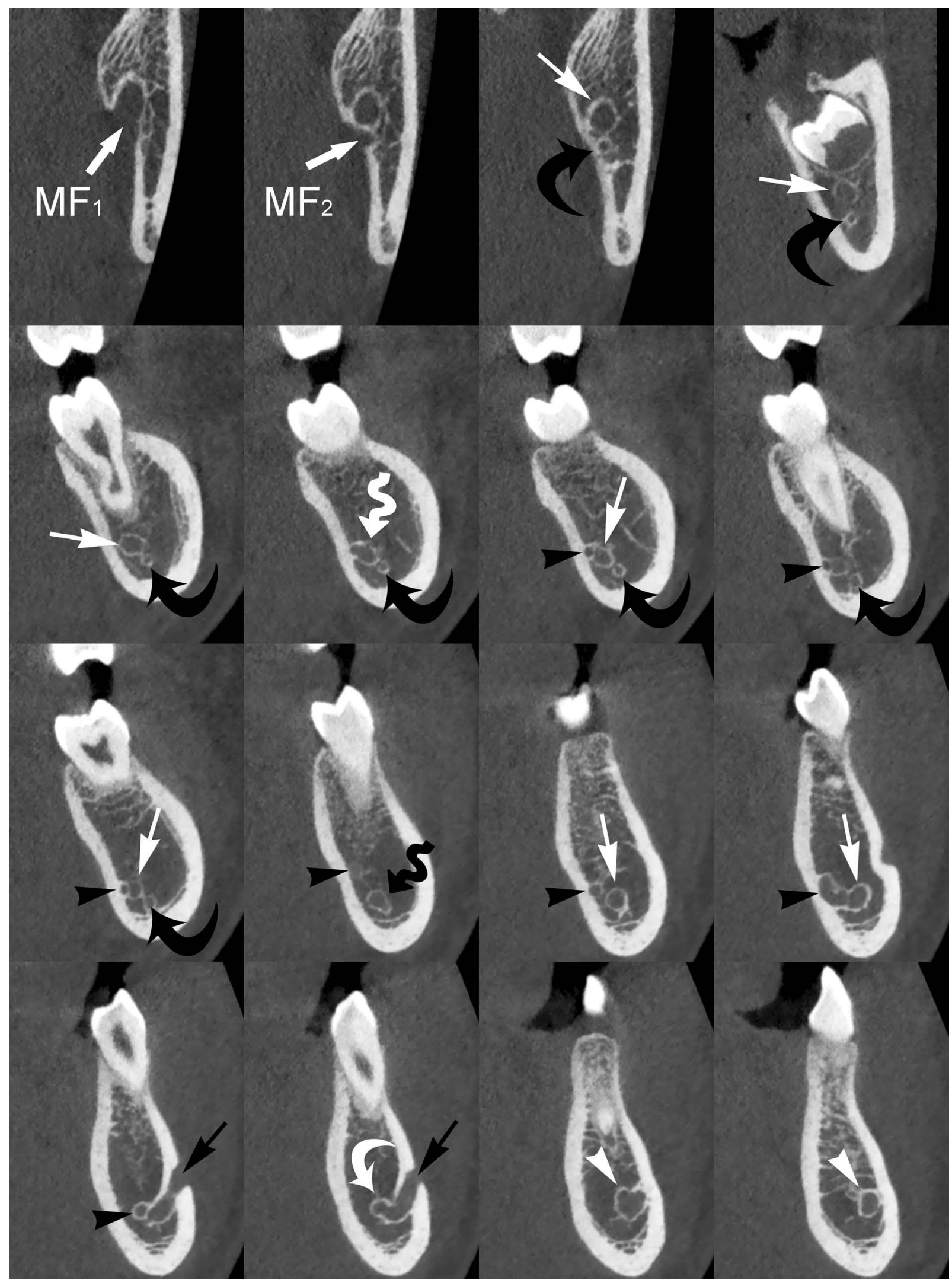

Figure 1. Cross-sectional cone-beam computed tomography images show the buccolingual position of the three mandibular canals (MC $A M C_{1}, A M C_{2}$ ) on the left hemimandible. $A M C_{1}$ (black arrowheads) originates from $M_{\text {main }}$ at the level of the second molar (white wavy arrow). $A M C_{1}$ joins $\mathrm{MC}_{\text {main }}$ at the level of the mental foramen (white curved arrow). $A M C_{2}$ (black curved arrows) originates from a small accessory mandibular foramen $\left(\mathrm{MF}_{2}\right)$ inferior to the main mandibular foramen $\left(\mathrm{MF}_{1}\right) . \mathrm{AMC}_{2}$ joins $\mathrm{MC}_{\text {main }}$ at the level of the second premolar (black wavy arrow); white arrows — $\mathrm{MC}_{\text {main }}$; white arrowheads — mandibular incisive canal; black arrows — mental foramen. 


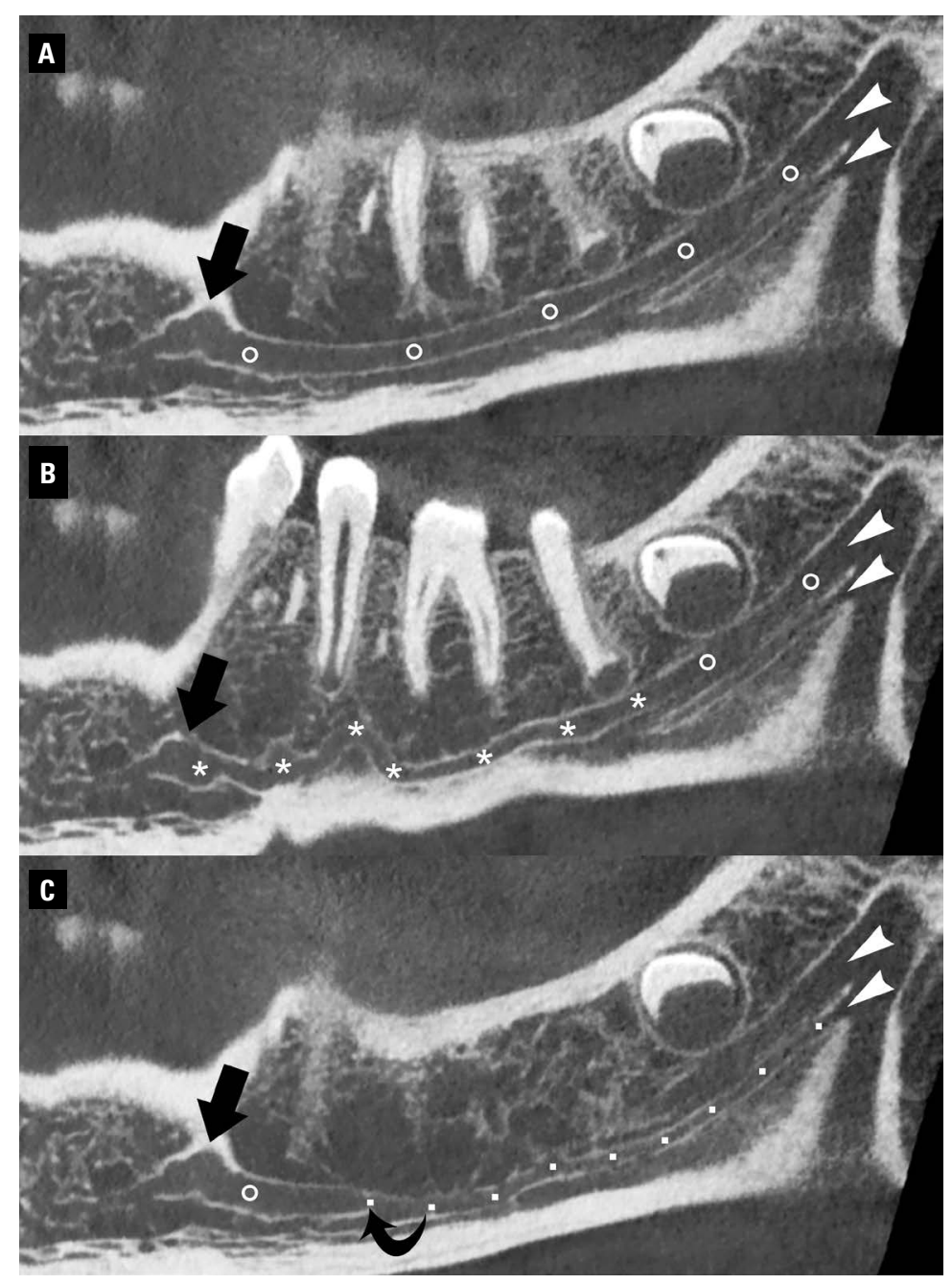

Figure 2. Panoramic cone-beam computed tomography images demonstrate the distomesial course of the three mandibular canals $\left(M C_{\text {main }}\right.$ $A M C_{1}, A M C_{2}$ ) within the left hemimandible; $\mathbf{A}, \mathbf{C}$. The intra-mandibular course of $\mathrm{MC}_{\text {main }}$ (circles) and $\mathrm{AMC}_{2}$ (dots) forms an upward-facing curve. $\mathrm{AMC}_{2}$ joins $\mathrm{MC}_{\text {main }}$ at the level of the second premolar (curved arrow); $\mathbf{B}$. The intra-mandibular course of $\mathrm{AMC}_{1}$ (asterisks) shows a serpiginous shape in its anterior portion. $A M C_{1}$ originated from $\mathrm{MC}_{\text {main }}$ at the level of the second molar. Double mandibular foramen is also shown (arrowheads); large black arrows - mental foramen.

To the best of our knowledge, the current report describes the CBCT findings of a novel anatomical variant characterised by the presence of a triple mandibular canal associated with a double mandibular foramen; a variant that is not included in the classification systems of mandibular canal branching $[2,11]$, and that has not been reported previously.

Differently from bifid and trifid mandibular canals $[12,14]$ which are confined in the retromolar or molar region, our accessory mandibular canals AMC $_{1}$ and $\mathrm{AMC}_{2}$ ) ran forward and continued their intra-mandibular course beyond the posterior mandible by reaching the premolar region (Fig. 2). AMC $M C_{\text {main }}$ at the level of the mental foramen, whereas $\mathrm{AMC}_{2}$ joined $\mathrm{MC}_{\text {main }}$ at the level of the second premolar (Figs. 1, 2).
From a clinical point of view, accessory mandibular canals and foramina have relevant clinical implications because they can increase the risk of neurovascular complications and inadequate local anaesthesia during mandibular surgical procedures. Therefore, preoperative radiological analysis of mandibular anatomical landmarks and their variations is of great importance to determine appropriate clinical management and reduce the occurrence of iatrogenic complications and IAN block failures.

In clinical practice, the presence of one or more accessory mandibular canals and/or foramina may be detected with different radiological techniques such as conventional $\mathrm{X}$-rays, multidetector $\mathrm{CT}$ and $\mathrm{CBCT}$. The main limitations of conventional $X$-rays (periapical and panoramic radiographs) include the two-dimen- 
sional nature of these radiographic examinations and the overlap of adjacent anatomical structures due to the lack of any cross-sectional information. Given these limitations, certain anatomical variants within mandible may be difficult to detect. In addition, the presence of these variants could sometimes be misinterpreted as osteolytic lesion on conventional $\mathrm{X}$-rays.

Both multidetector CT and CBCT are usually adequate for radiological assessment of the mandible. However, it is well known that CBCT outperform multidetector $\mathrm{CT}$ in displaying fine anatomical details (such as canals and foramina). Therefore, CBCT is currently considered the most accurate radiological imaging technique to study of fine anatomical details of the mandible and its variants.

With the introduction and broad availability of CBCT scanners in clinical practice, an increasing number of anatomical variants are being detected, especially in the mandibular region [7, 9]. The number of reports that describe unusual anatomical variants within the mandible is also steadily increasing $[1,4,5]$. Therefore, radiologists should be aware of the clinical importance of reporting the number, size and course of any accessory mandibular canals and any additional variants that are incidentally observed during CBCT examinations.

\section{CONCLUSIONS}

This report describes the novel presentation of a triple mandibular canal associated with double mandibular foramen in a living human subject. Given that the occurrence of critical anatomical variants associated with an increased risk of iatrogenic injury of the IAN is becoming more frequent nowadays, a preoperative $\mathrm{CBCT}$ study of the mandible is recommended before any oral and dental procedures are carried out in the mandibular region. In addition, awareness about the presence of these critical anatomical variants can also help prevent future misdiagnosis and unnecessary radiological investigations.

\section{REFERENCES}

1. Borghesi A, Pezzotti S, Nocivelli G, et al. Five mental foramina in the same mandible: CBCT findings of an unusual anatomical variant. Surg Radiol Anat. 2018; 40(6): 635-640, doi: 10.1007/s00276-018-1969-5, indexed in Pubmed: 29318364.

2. Castro MA, Lagravere-Vich MO, Amaral TM, et al. Classifications of mandibular canal branching: A review of literature. World J Radiol. 2015; 7(12): 531-537, doi: 10.4329/wjr.v7.i12.531, indexed in Pubmed: 26753068.
3. Chávez-Lomeli ME, Mansilla Lory J, Pompa JA, et al. The human mandibular canal arises from three separate canals innervating different tooth groups. J Dent Res. 1996; 75(8): 1540-1544, doi: 10.1177/00220345960750080401, indexed in Pubmed: 8906121.

4. Choi YY, Han SS. Double mandibular foramen leading to the accessory canal on the mandibular ramus. Surg Radiol Anat. 2014; 36(9): 851-855, doi: 10.1007/s00276-0141310-x, indexed in Pubmed: 24817561.

5. de Souza Tolentino E, Silva PA, Pagin O, et al. Uncommon trajectory variations of the mandibular canal and of the mandibular incisive canal: case report. Surg Radiol Anat. 2013; 35(9): 857-861, doi: 10.1007/s00276-013-1138-9, indexed in Pubmed: 23728516.

6. Fuentes R, Arias A, Farfán C, et al. Morphological variations of the mandibular canal in digital panoramic radiographs: a retrospective study in a Chilean population. Folia Morphol. 2019; 78(1): 163-170, doi: 10.5603/FM.a2018.0058, indexed in Pubmed: 30009366.

7. Kawai T, Sato I, Asaumi R, et al. Cone-beam computed tomography and anatomical observations of normal variants in the mandible: variant dentists should recognize. Oral Radiol. 2018; 34(3): 189-198, doi: 10.1007/s11282-0170307-7, indexed in Pubmed: 30484034.

8. Kqiku L, Weiglein AH, Pertl C, et al. Histology and intramandibular course of the inferior alveolar nerve. Clin Oral Investig. 2011; 15(6): 1013-1016, doi: 10.1007/s00784010-0459-x, indexed in Pubmed: 20737177.

9. Leite GM, Lana JP, de Carvalho Machado V, et al. Anatomic variations and lesions of the mandibular canal detected by cone beam computed tomography. Surg Radiol Anat. 2014; 36(8): 795-804, doi: 10.1007/s00276-013-1247-5, indexed in Pubmed: 24337387.

10. Muinelo-Lorenzo J, Suárez-Quintanilla JA, Fernández-Alonso A, et al. Descriptive study of the bifid mandibular canals and retromolar foramina: cone beam CT vs panoramic radiography. Dentomaxillofac Radiol. 2014; 43(5): 20140090, doi: 10.1259/dmfr.20140090, indexed in Pubmed: 24785820.

11. Ngeow WC, Chai WL, Ngeow WC, et al. The clinical anatomy of accessory mandibular canal in dentistry. Clin Anat. 2020; 33(8): 1214-1227, doi: 10.1002/ca.23567, indexed in Pubmed: 31943382.

12. Rashsuren O, Choi JW, Han WJ, et al. Assessment of bifid and trifid mandibular canals using cone-beam computed tomography. Imaging Sci Dent. 2014; 44(3): 229-236, doi: 10.5624/isd.2014.44.3.229, indexed in Pubmed: 25279344.

13. Rodella LF, Buffoli B, Labanca M, et al. A review of the mandibular and maxillary nerve supplies and their clinical relevance. Arch Oral Biol. 2012; 57(4): 323-334, doi: 10.1016/j. archoralbio.2011.09.007, indexed in Pubmed: 21996489.

14. Yang X, Lyu C, Zou D. Bifid mandibular canals incidence and anatomical variations in the population of Shanghai area by cone beam computed tomography. J Comput Assist Tomogr. 2017; 41(4): 535-540, doi: 10.1097/ RCT.0000000000000561, indexed in Pubmed: 28722697.

15. Zhang YQ, Zhao YN, Liu DG, et al. Bifid variations of the mandibular canal: cone beam computed tomography evaluation of 1000 Northern Chinese patients. Oral Surg Oral Med Oral Pathol Oral Radiol. 2018; 126(5): e271-e278, doi: 10.1016/j. oooo.2018.06.008, indexed in Pubmed: 30093317. 